\title{
Time-dependent electron tunneling through time-dependent tunnel barriers
}

\author{
Z. S. Gribnikov \\ Institute for Quantum Sciences, Michigan State University, East Lansing, Michigan 48824 \\ and Department of EECS, University of Michigan, Ann Arbor, Michigan 48109 \\ G. I. Haddad \\ Department of EECS, University of Michigan, Ann Arbor, Michigan 48109
}

(Received 9 January 2004; accepted 22 June 2004)

\begin{abstract}
A plane electron wave incident on a tunnel-transparent potential barrier formed by the potential $V(x, t)=V_{0}(x)+V_{1}(x) \cos \omega t$ generates, in addition to the usual stationary transmitted and reflected stationary waves, also "transmitted" and "reflected" electron waves oscillating with the same frequency $\omega$. The transmitted oscillating wave can serve as the basis for transit-time microwave generators oscillating in the terahertz range. (Such oscillators are ballistic analogs of the tunnel-emission transit-time diode oscillators suggested almost half a century ago.) In the special case of a rectangular potential barrier, we describe the dependence of a small transmitted oscillating wave amplitude on the frequency $\omega$ and the value of $V_{1}(x)$. We consider two forms of $V_{1}(x)$ : (1) homogeneous oscillation of the height of the rectangular barrier and (2) $V_{1}(x)=a \delta\left(x-x_{1}\right)$ [where $\delta(x)$ is the Dirac delta function and $0<x_{1}<w ; w$ is the barrier thickness]. For sufficiently high frequencies $\omega$ determined by the time for tunneling, a much higher emission of the transmitted oscillating wave takes place in comparison with the results of quasistatic calculations. (C) 2004 American Institute of Physics. [DOI: 10.1063/1.1783592]
\end{abstract}

\section{INTRODUCTION}

Starting from the pioneering work of Keldysh, ${ }^{1}$ electron tunneling through nonstationary barriers has been the subject of inexhaustible scientific interest. The classic problem of such a type is a tunnel ionization of a neutral atom in a homogeneous alternating electric field ${ }^{1,2} \mathcal{E}=\mathcal{E}_{1} \cos \omega t$. If the frequency $\omega$ is sufficiently low, the ionization rate does not depend on $\omega$ and is determined only by the field amplitude $\mathcal{E}_{1}$. This rate can be calculated on the basis of static tunneling theory. An increase in $\omega$ induces dispersion and a characteristic frequency that defines this dispersion is $\omega \cong \omega_{C}$ $=e \mathcal{E}_{1} / \sqrt{2 m \delta}$ where $m$ is an electron mass (or effective mass) and $\delta$ is the ionization energy. A simple evaluation shows that for $\mathcal{E}_{1} \sim 10^{4}-10^{5} \mathrm{~V} / \mathrm{cm}$ and $\delta \sim 0.1 \mathrm{eV}$, the characteristic frequency $\omega_{C}$ is in the terahertz $(\mathrm{THz})$ range.

The other classic problem ${ }^{3}$ deals with tunneling in the case of the combined action of static and alternating electric fields when a time-dependent barrier can be described by the electric potential $V(x)=V_{0}(x)+V_{1}(x) \cos \omega t$, where $V_{1}(x)$ describes a small periodic modulation of the static potential $V_{0}(x)$. The tunneling through such a barrier can be expressed in terms of emission or absorption of radiation quanta $\hbar \omega$ and the probabilities of these processes depend on $\omega$ substantially. The probability of the quantum absorption, which drastically magnifies the full tunneling probability, becomes noticeable beginning at $\omega_{T}=2 \pi / \tau_{T}$, where $\tau_{T}$ is the traversal time for tunneling evaluated by the quasiclassical formula ${ }^{3,4}$

$$
\tau_{T}=\int_{x_{1}}^{x_{2}}\left\{m / 2\left[e V_{0}(x)-\varepsilon\right]\right\}^{1 / 2} d x
$$

In Eq. (1) $\varepsilon$ is the energy of a tunneling electron, and $x_{1}$ and $x_{2}$ are turning points. As is seen in Eq. (1), $\tau_{T}$ is determined only by parameters of the static barrier.
The dependence of the tunneling probability on all the parameters of the potential $V_{1}(x)$ is considered in great detail in Refs. 5 and 6. In Ref. 6, it is not assumed that the potential $V_{1}(x)$ is known to be small. Therefore, an increase in the alternating electric field amplitude induces multiquantum processes with the absorption of larger than one radiation quanta (see also Ref. 3).

Note also the appearance of the extensive cycle of experimental results ${ }^{7-10}$ (see also references in Ref. 10) regarding the ionization of deep centers in semiconductors (AlGaAs, AlGaSb, Ge) in $\mathrm{THz}$ electric fields ( $\omega$ $=3.4-200 \mathrm{THz}$ ). In the same cycle, a theory of a phononassisted tunneling in high-frequency fields (taking place in the experiments) is substantially developed.

Our interest in tunneling through time-dependent barriers is connected with a possible promotion of the so-called tunnett diodes to the $\mathrm{THz}$ range. These diodes are transittime (TT) microwave generators with a tunneling electron emission into a transit space (the acronym TUNNETT means TUNNel emission transit time). Among the family of TT generators, only tunnett diodes could be designed in a completely ballistic version. Therefore these diodes are potentially the highest-frequency generators. [The family of TT generators also includes IMPATT (IMPact ionization avalanche transit time), BARITT (BARrier injection transit time), TRAPATT (TRApped plasma avalanche triggered transit) diodes, ${ }^{11,12}$ etc. All of these diodes cannot be ballistic in principle.] The $\mathrm{THz}$ promotion of tunnett diodes has been demonstrated in recent experiments. ${ }^{13}$ Also, the theory of ballistic TT diodes with a tunnel electron emission has been developed recently. ${ }^{14-16}$ Such diodes can be designed as $\mathrm{THz}$ oscillators. 


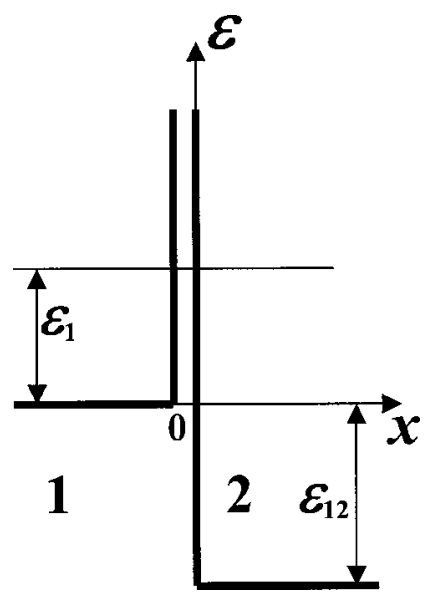

FIG. 1. $\delta$-function potential barrier.

As is known, the static transparence of a tunnel barrier with the potential $V_{0}(x)$ is described by the approximate formula

$$
D \approx \exp \left\{-(2 / \hbar) \int_{x_{1}}^{x_{2}} \sqrt{2 m\left[e V_{0}(x)-\varepsilon\right]} d x\right\},
$$

which is convenient to compare with Eq. (1). The same values of $D$ can be obtained by different parameter versions leading to different values of $\tau$ in Eq. (1). In the case of a rectangular barrier, the $n^{2}$ times increase in its "height" $\left(V_{0}\right.$ $-\varepsilon)$, simultaneous with the $n$ times decrease in its length $x_{2}-x_{1}$, protects values of $D$ (and a direct current of electrons with energy $\varepsilon$ ), but the time for tunneling $\tau_{T}$ decreases $n^{2}$ times. This means that such a varied barrier should be quasistatic up to much higher frequencies of a small barrier potential addition $V_{1}(x)$.

Below, in Sec. III, we consider the frequency and amplitude dependences of an electron tunnel transparence of a rectangular potential barrier with thickness $w$ whose height oscillates periodically with the frequency $\omega$ and the amplitude $e V_{1}$. We compare the results of quasistatic calculations with the results of a consistent nonstationary theory. It is shown that an increase in frequency leads to a substantial increase in the amplitude of a high-frequency current for a certain amplitude $e V_{1}$. This consideration follows the theory of a time-dependent $\delta$-function barrier ( $\delta$ barrier), which is always quasistatic, in Sec. II. In Sec. IV, we consider the same rectangular potential barrier, as in Sec. III, perturbed by the time-dependent $\delta$ barrier placed in some arbitrary position $x_{1}\left(0 \leqslant x_{1} \leqslant w\right)$. The obtained results are discussed in Sec. V and summarized in Sec. VI.

\section{TUNNELING THROUGH A $\delta$-FUNCTION BARRIER} form

In this section, we consider a barrier potential in the

$$
e V(x, t)=(A+a \cos \omega t) \delta(x),
$$

where $\delta(x)$ is the Dirac delta function. This potential is localized on the border between two homogeneous regions 1 and 2, shown in Fig. 1. The bottoms of the conduction bands do not coincide with each other: there is a discontinuity $\varepsilon_{12}$. Effective masses are also different: $m_{1} \neq m_{2}$.

Let us consider the wave function of an electron incident from the left on the tunnel barrier with energy $\varepsilon_{1}$ $=\hbar^{2} k_{1}^{2} / 2 m_{1}$, and then partially reflecting back and partially passing to region 2 . It is described by the Schrödinger equation:

$$
\begin{aligned}
i \hbar \frac{\partial \Psi_{1,2}}{\partial t}= & -\frac{\hbar^{2}}{2 m_{1,2}} \frac{\partial^{2} \Psi_{1,2}}{\partial x^{2}}+(A+a \cos \omega t) \delta(x) \Psi_{1,2} \\
& -\varepsilon_{12} \theta(x) \Psi_{2},
\end{aligned}
$$

where $\theta(x)=0$ for $x<0$ and $\theta(x)=1$ for $x>0$. Functions $\Psi_{1,2}(x)$ in the homogeneous regions 1 and 2 are joined together by the boundary conditions:

$$
\Psi_{1}(-0)=\Psi_{2}(+0)=\Psi(0)
$$

and

$$
\begin{aligned}
& \left(\hbar^{2} / 2\right)\left[\left.\left(1 / m_{2}\right)\left(\partial \Psi_{2} / \partial x\right)\right|_{+0}-\left.\left(1 / m_{1}\right)\left(\partial \Psi_{1} / \partial x\right)\right|_{-0}\right] \\
& \quad=(A+a \cos \omega t) \Psi(0) .
\end{aligned}
$$

The solutions of Eq. (4) on both sides of the barrier can be written in the following form:

$$
\begin{aligned}
\Psi_{1}(x, t)= & \exp \left(-i \Omega_{1} t\right)\left[\exp \left(i k_{1} x\right)+B_{0} \exp \left(-i k_{1} x\right)\right. \\
& +B_{+} \exp \left(-i k_{(1+)} x+i \omega t\right)+B_{-} \exp \left(-i k_{(1-)} x\right. \\
& -i \omega t)], \\
\Psi_{2}(x, t)= & \exp \left(-i \Omega_{1} t\right)\left[F_{0} \exp \left(i k_{2} x\right)+F_{+} \exp \left(i k_{(2+)} x\right.\right. \\
& \left.+i \omega t)+F_{-} \exp \left(i k_{(2-)} x-i \omega t\right)\right],
\end{aligned}
$$

where $\Omega_{1}=\varepsilon_{1} / \hbar, \quad k_{1}^{2}=2 m_{1} \Omega_{1} / \hbar, \quad k_{2}^{2}=2 m_{2} \Omega_{2} / \hbar, \quad \Omega_{2}=\Omega_{1}$ $+\varepsilon_{12} / \hbar, \quad k_{(1 \pm)}^{2}=2 m_{1}\left(\Omega_{1} \mp \omega\right) / \hbar$, and $k_{(2 \pm)}^{2}=2 m_{2}\left(\Omega_{2} \mp \omega\right) / \hbar$. Using the "boundary" conditions (5) and (6), we obtain

$$
\begin{aligned}
& F_{0}=-2 i k_{1} /\left[m_{1}(\kappa-i \lambda)\right], \\
& B_{0}=-\left(1-F_{0}\right)=-\left\{\kappa-i\left[\left(k_{2} / m_{2}\right)-\left(k_{1} / m_{1}\right)\right]\right\} /(\kappa-i \lambda),
\end{aligned}
$$

where

$$
\kappa=2 A / \hbar^{2}, \lambda=\left(k_{1} / m_{1}\right)+\left(k_{2} / m_{2}\right),
$$

and

$$
B_{ \pm}=F_{ \pm}=-i \chi F_{0} /\left[2\left(\kappa-i \lambda_{ \pm}\right)\right],
$$

where

$$
\chi=2 a / \hbar^{2}, \lambda_{ \pm}=\left(k_{(1 \pm)} / m_{1}\right)+\left(k_{(2 \pm)} / m_{2}\right) .
$$

An electron current (with the velocity dimension) corresponding to the wave functions (7) and (8) and calculated with assumption $|a| \ll A$ consists of the stationary and homogeneous component,

$$
\begin{aligned}
j_{0} & =\hbar k_{2}\left|F_{0}\right|^{2} / m_{2}=\hbar k_{1}\left(1-\left|B_{0}\right|^{2}\right) \\
& =4 \hbar k_{2} k_{1}^{2} / m_{2} m_{1}^{2}\left(\kappa^{2}+\lambda^{2}\right),
\end{aligned}
$$

and the inhomogeneous and nonstationary periodic component. We write the latter on both sides of the barrier: 


$$
\begin{aligned}
j_{1}(x, t)= & -\left(\hbar / 2 m_{1}\right)\left(( k _ { 1 } + k _ { ( 1 + ) } ) \left\{B _ { 0 } B _ { + } ^ { * } \operatorname { e x p } \left[-i\left(k_{1}-k_{(1+)}\right) x\right.\right.\right. \\
& -i \omega t]+ \text { c.c. }\}+\left(k_{1}+k_{(1-)}\right)\left\{B _ { 0 } B _ { - } ^ { * } \operatorname { e x p } \left[-i\left(k_{1}\right.\right.\right. \\
& \left.\left.\left.\left.-k_{(1-)}\right) x+i \omega t\right]+ \text { c.c. }\right\}\right)+\left(\hbar / 2 m_{1}\right)\left(\left(k_{1}-k_{(1+)}\right)\right. \\
& \times\left\{B_{+} \exp \left[-i\left(k_{1}+k_{(1+)}\right) x+i \omega t\right]+\text { c.c. }\right\}+\left(k_{1}\right. \\
& \left.\left.-k_{(1-)}\right)\left\{B_{-} \exp \left[-i\left(k_{1}+k_{(1-)}\right) x-i \omega t\right]+\text { c.c. }\right\}\right),
\end{aligned}
$$

$$
\begin{aligned}
j_{2}(x, t)= & \left(\hbar / 2 m_{2}\right)\left(( k _ { 2 } + k _ { ( 2 + ) } ) \left\{F _ { 0 } F _ { + } ^ { * } \operatorname { e x p } \left[i\left(k_{2}-k_{(2+)}\right) x\right.\right.\right. \\
& -i \omega t]+ \text { c.c. }\}+\left(k_{2}+k_{(2-)}\right)\left\{F _ { 0 } F _ { - } ^ { * } \operatorname { e x p } \left[i \left(k_{2}\right.\right.\right. \\
& \left.\left.\left.\left.-k_{(2-)}\right) x+i \omega t\right]+ \text { c.c. }\right\}\right),
\end{aligned}
$$

where c.c. means a complex conjugated expression. First, we consider Eq. (11) that describes a "transmitted" alternating current on the right side. For $\omega \ll \Omega_{2}$ when $k_{2}-k_{(2-)} \cong-\left(k_{2}\right.$ $\left.-k_{(2+)}\right) \cong-k_{2} \omega / 2 \Omega_{2}, \quad k_{2}+k_{(2 \pm)} \cong 2 k_{2}, \quad \lambda_{ \pm} \cong \lambda$, and $F_{+} \cong F_{-}$ $\cong i \chi k_{1} / 2(\kappa-i \lambda)^{2}$, we have

$$
\begin{aligned}
j_{2}(x, t) \cong & -8 \hbar\left(k_{2} / m_{2}\right)\left(k_{1} / m_{1}\right)^{2}\left[\chi \kappa /\left(\kappa^{2}+\lambda^{2}\right)^{2}\right] \\
& \times \cos \left[(1 / 2) k_{2}\left(\omega / \Omega_{2}\right) x-\omega t\right] \\
= & -\left[2 \chi \kappa /\left(\kappa^{2}+\lambda^{2}\right)\right] j_{0} \cos \left[\left(k_{2} \omega x / 2 \Omega_{2}\right)-\omega t\right] .
\end{aligned}
$$

So, the current $j_{2}(x, t)$ is an oscillating wave with the wavelength

$$
\Lambda=4 \pi \Omega_{2} / k_{2} \omega .
$$

The length $\Lambda$ exceeds $2 \Omega_{2} / \omega$ times the electron wavelength $2 \pi / k_{2}$. Since the alternating component of the $\delta$-barrier potential is proportional to $\cos \omega t$, the current $j_{2}(x, t)$ leads the barrier potential by $2 \pi x / \Lambda$ in phase.

Note that an expression for $j_{2}(0, t)$ can be obtained directly from Eq. (9) if it is replaced on the right-hand side, $\kappa^{2} \rightarrow(\kappa+\chi \cos \omega t)^{2} \cong \kappa^{2}+2 \kappa \chi \cos \omega t$, and the smallness of the second component is taken into account.

The alternating current on the left side $(x<0)$ presented by Eq. (10) consists of two components. The first of them, expressed by the first two and half lines on the right-hand side of Eq. (10), is a "reflected" alternating current wave. The structure of this component, $j_{1}^{\prime}(x, t)$, is close to the structure of the considered transmitted wave (11). They differ from each other mainly by wave vector values and directions. Taking into account that $\omega \ll \Omega_{1}$, we obtain

$$
\begin{aligned}
j_{1}^{\prime}(x, t) \cong & -4 \hbar\left(k_{1} / m_{1}\right)^{2}\left[\chi \kappa \lambda /\left(\kappa^{2}+\lambda^{2}\right)^{2}\right] \\
& \times \cos \left[(1 / 2) k_{1}\left(\omega / \Omega_{1}\right) x+\omega t\right] \\
=- & {\left[\chi \kappa \lambda /\left(k_{2} / m_{2}\right)\left(\kappa^{2}+\lambda^{2}\right)\right] j_{0} } \\
& \times \cos \left[\left(k_{1} \omega x / 2 \Omega_{1}\right)+\omega t\right] .
\end{aligned}
$$

The last two and half lines on the right-hand side of Eq. (10) present the high-frequency reflected current wave with very short wavelength (twice shorter than the initial electron wavelength). The amplitude of this wave is proportional to $\omega / \Omega_{1}$ and small in comparison with the $j_{1}^{\prime}(x, t)$ amplitude. Comparing the amplitudes of the currents $j_{1}(x, t) \cong j_{1}^{\prime}(x, t)$ and $j_{2}(x, t)$, which relate to each other as $\left|j_{1 \max }^{\prime} / j_{2 \max }\right|$

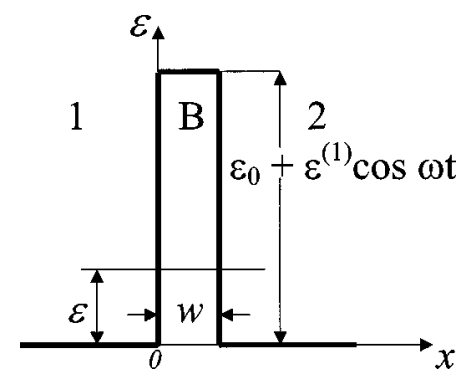

FIG. 2. Homogeneous rectangular potential barrier with the time-dependent height.

$=\left[\left(k_{1} / m_{1}\right)+\left(k_{2} / m_{2}\right)\right] / 2\left(k_{2} / m_{2}\right)$, we can see that the reflected wave knowingly exceeds the transmitted one if $\left(k_{1} / m_{1}\right)$ $\gg\left(k_{2} / m_{2}\right)$ and is only twice smaller than the transmitted wave in the opposite case.

In the symmetric case when $m_{1}=m_{2}=m, \varepsilon_{12}=0, \Omega_{1}$ $=\Omega_{2}=\Omega$, and $k_{1}=k_{2}=k$, formulas (12) and (14) are noticeably simplified:

$$
\begin{aligned}
j_{2}(x, t) \cong & -8 \hbar(k / m)^{3}\left[\chi \kappa /\left(\kappa^{2}+\lambda^{2}\right)^{2}\right] \\
& \times \cos [(1 / 2) k(\omega / \Omega) x-\omega t], \\
j_{1}^{\prime}(x, t) \cong & -8 \hbar(k / m)^{3}\left[\chi \kappa /\left(\kappa^{2}+\lambda^{2}\right)^{2}\right] \\
& \times \cos [(1 / 2) k(\omega / \Omega) x+\omega t] .
\end{aligned}
$$

\section{TUNNELING THROUGH A RECTANGULAR BARRIER WITH A TIME-DEPENDENT HEIGHT}

In the previous example, the high-frequency modulation of the $\delta$-barrier transparence has led to the high-frequency modulation of the reflected and transmitted current oscillating waves, but the modulation depth has been independent of the frequency (at least, for $\omega \ll \Omega_{1,2}$ ). The introduced parameter $\chi=2 a / \hbar^{2}$ does not contain any evident frequency dispersion. In this section, we consider one more model example: we replace the $\delta$ barrier with a rectangular potential barrier (Fig. 2) with the unvaried thickness $w$ and the timedependent height experiencing a small modulation:

$$
\varepsilon_{B}=\varepsilon_{0}+\varepsilon^{(1)} \cos \omega t .
$$

To simplify, we consider only the symmetric situation: $m_{1}$ $=m_{2}=m, \hbar \Omega_{1}=\hbar \Omega_{2}=\hbar \Omega=\varepsilon<\varepsilon_{0}-\varepsilon^{(1)}$. We assume effective masses inside and outside are equal: $m_{B}=m$. Therefore, we sew both wave functions and their derivatives in both boundaries of the barrier $(x=0, x=w)$. In fact, we solve the problem of a rectangular barrier in a homogeneous medium. The solutions of the Schrödinger equation on both side of the barrier are written by formulas (7) and (8) where $\Omega_{1}=\Omega_{2}=\Omega$ $=\varepsilon / \hbar, k_{1}=k_{2}=k$, and $k_{(1 \pm)}=k_{(2 \pm)}=k_{ \pm}$. To obtain correlations between $B_{0}, B_{ \pm}$and $F_{0}, F_{ \pm}$, we need to solve the Schrödinger equation in the barrier:

$$
i \hbar \frac{\partial \Psi_{B}}{\partial t}=-\frac{\hbar^{2}}{2 m} \frac{\partial^{2} \Psi_{B}}{\partial x^{2}}+\left(\varepsilon_{0}+\varepsilon^{(1)} \cos \omega t\right) \Psi_{B} .
$$

Its solution for $\varepsilon^{(1)}=0$ is 


$$
\Psi_{B}(x, t)=\exp (-i \Omega t)\left[X_{1} \exp (-g x)+X_{2} \exp (g x)\right]
$$

where $g=(1 / \hbar) \sqrt{2 m\left(\varepsilon_{0}-\varepsilon\right)}$. Using solution (17), we obtain

$$
\begin{aligned}
& j_{0}=(\hbar k / m)\left|F_{0}\right|^{2}=(\hbar k / m)\left(1-\left|B_{0}\right|^{2}\right)=4(\hbar k / m) \mu^{2}\left|\Delta_{0}\right|^{-2}, \\
& X_{1}=-i \mu(1-i \mu) \Delta_{0}^{-1} \exp (g w), \\
& X_{2}=-i \mu(1+i \mu) \Delta_{0}^{-1} \exp (-g w), F_{0}=-2 i \mu \Delta_{0}^{-1}, \\
& B_{0}=-\left(1+\mu^{2}\right) \Delta_{0}^{-1} \sinh g w,
\end{aligned}
$$

where

$$
\begin{aligned}
& \mu=k / g, \Delta_{0}=\left(1-\mu^{2}\right) \sinh g w-2 i \mu \cosh g w, \\
& \left|\Delta_{0}\right|^{2}=\left(1-\mu^{2}\right)^{2} \sinh ^{2} g w+4 \mu^{2} \cosh ^{2} g w .
\end{aligned}
$$

Equation (18) can be used to obtain a small and comparatively low-frequency alternating current $j_{2}(0, t)$ analogous to the preceding section. Assuming that a quasistatic process occurs for a low frequency $\omega \ll \Omega$, we can replace $\varepsilon_{0}-\varepsilon$ in Eq. (18) with $\varepsilon_{0}-\varepsilon+\varepsilon^{(1)} \cos \omega t$ (in the expressions for $g$ ). Such a replacement in the case of the assumed smallness of $\varepsilon^{(1)}$ leads to the following formula:

$$
\begin{aligned}
j_{2}(0, t)= & -\left[\frac{\left(1-\mu^{2}\right)(\cosh 2 g w-1)+\left(1+\mu^{2}\right) \sinh 2 g w}{2\left|\Delta_{0}\right|^{2}}\right] \\
& \times \frac{\left(1+\mu^{2}\right) \varepsilon^{(1)}}{\varepsilon_{0}-\varepsilon} j_{0} \cos \omega t
\end{aligned}
$$

where $j_{0}$ is defined by Eq. (18). Since Eq. (19) structurally resembles Eq. (12), we can try to tie them together assuming that $g w \ll 1$ (thin barrier) and $\varepsilon_{0} \gg \varepsilon$ (high barrier). Identity takes place if $A=\hbar^{2} \kappa / 2=\varepsilon_{0} w$ and $a=\hbar^{2} \chi / 2=\varepsilon^{(1)} w$. In the case of a thin barrier $(g w \ll 1)$, a condition of smallness of the barrier height oscillation amplitude,

$$
\varepsilon^{(1)} \ll \varepsilon_{0}-\varepsilon,
$$

is sufficient to obtain Eq. (19). But in the case of thicker barriers $(2 g w \geqslant 1)$, comparatively little height oscillations can lead to nonlinear distortions of a quasistatic current $j_{2}(0, t)$. Therefore, Eq. (19) is justified only if

$$
\varepsilon^{(1)} \ll\left(\varepsilon_{0}-\varepsilon\right) / 2 g w .
$$

Disruption of the condition (21) leads to the appearance of numerous harmonics in both transmitted and reflected currents.

Now let us reject the quasistatic approach, which has been used only to obtain the illustrative formula (19), and let us try to find the solution of Eq. (16) suitable for any reasonable value of $\omega$. Separating variables in Eq. (16), we obtain in the barrier

$$
\begin{aligned}
\Psi_{B}(\varepsilon ; x, t)= & {\left[X_{1} \exp (-i g x)+X_{2} \exp (g x)\right] \exp [-(i \varepsilon t / \hbar)} \\
& \left.-\left(i \omega_{1} / \omega\right) \sin \omega t\right]
\end{aligned}
$$

with $g^{2}=2 m\left(\varepsilon_{0}-\varepsilon\right) / \hbar^{2}$ as before and $\omega_{1}=\varepsilon^{(1)} / \hbar$. In the simplest case when the barrier height pulsation frequency $\omega$ is sufficiently high,

$$
\omega \gg \omega_{1}=\varepsilon^{(1)} / \hbar,
$$

the solution (22) can be rewritten in the approximate form as follows:

$$
\begin{aligned}
\Psi_{B}(\varepsilon ; x, t) \cong & {\left[X_{1} \exp (-i g x)+X_{2} \exp (g x)\right] } \\
& \times \exp (-i \varepsilon t / \hbar)(1-2 i \alpha \sin \omega t) \\
= & {\left[X_{1} \exp (-i g x)+X_{2} \exp (g x)\right] } \\
& \times\{\exp (-i \Omega t)-\alpha \exp [-i(\Omega-\omega) t] \\
& +\alpha \exp [-i(\Omega+\omega) t]\},
\end{aligned}
$$

where $\alpha=\omega_{1} / 2 \omega$ and $\Omega=\varepsilon / \hbar$. Let us note that the strong inequality (23) compares the barrier height pulsation frequency $\omega$ with the amplitude $\omega_{1}=\varepsilon^{(1)} / \hbar$ of the same pulsations. This means that our subdivision of low and high frequencies depends on pulsation amplitudes.

The solution (23) [even in its simplest form (24)] demonstrates that the initial electron wave with energy $\varepsilon=\hbar \Omega$ during its attenuation in the barrier, the height of which oscillates with the frequency $\omega$, becomes multienergy., Alongside the initial wave, satellite waves appear with energies $\hbar(\Omega \pm \omega), \hbar(\Omega \pm 2 \omega)$, and so on. We should also take into account the fact of the existence of these waves among both reflected and transferred waves (in regions 1 and 2 in Fig. 2). Each of these waves in the barrier body should be presented by a solution of the Eq. (22) type with its own amplitudes $X_{1}^{( \pm n \omega)}$ and $X_{2}^{( \pm n \omega)}$. These satellite waves in the high-frequency limit (23) lose to the initial wave in intensity (by the factor $\left.\alpha^{n}\right)$. But waves with energy $\hbar(\Omega+n \omega)$ attenuate in the barrier substantially weaker than the initial wave attenuates (if an attenuation of the latter is substantial). Therefore, just these weaker-attenuating satellite waves can be dominating in the transferred current. ${ }^{6}$ Below we consider such a situation in the simplest case when Eq. (23) takes place.

Neglecting all the components of order $\alpha^{n}(n \geqslant 2)$, we write the solutions in all the three regions in Fig. 2:

$$
\begin{aligned}
\Psi_{B}(x, t)= & \left(X_{1} e^{-g x}+X_{2} e^{g x}\right)\{\exp (-i \Omega t)-\alpha \exp [-i(\Omega \\
& -\omega) t]+\alpha \exp [-i(\Omega+\omega) t]\}+\left[X_{1}^{+} \exp \left(-i g_{+} x\right)\right. \\
& \left.+X_{2}^{+} \exp \left(g_{+} x\right)\right] \exp [-i(\Omega-\omega) t] \\
& +\left[X_{1}^{-} \exp \left(-i g_{-} x\right)+X_{2}^{-} \exp \left(g_{-} x\right)\right] \\
& \times \exp [-i(\Omega+\omega) t], \\
\Psi_{1}(x, t)= & \exp (-i \Omega t)\left[\exp \left(i k_{1} x\right)+B_{0} \exp (-i k x)\right. \\
& \left.+B_{+} \exp \left(-i k_{+} x+i \omega t\right)+B_{-} \exp \left(-i k_{-} x-i \omega t\right)\right],
\end{aligned}
$$

$$
\begin{aligned}
\Psi_{2}(x, t)= & \exp (-i \Omega t)\left[F_{0} \exp (i k x)+F_{+} \exp \left(i k_{+} x+i \omega t\right)\right. \\
& \left.+F_{-} \exp \left(i k_{-} x-i \omega t\right)\right]
\end{aligned}
$$

with $g_{ \pm}^{2}=2 m\left(\varepsilon_{0}-\varepsilon \pm \hbar \omega\right) / \hbar^{2}=2 m\left(\Omega_{0}-\Omega \pm \omega\right) / \hbar$.

The solutions outside the barrier, Eqs. (26) and (27), are the same as Eqs. (7) and (8), but the solution inside the barrier, Eq. (25), is of the more complicated form. This fact is connected directly with the barrier height modulation. Sewing together wave functions and their derivatives inside 
and outside the barrier in the barrier boundaries for each of the three energies $\varepsilon, \varepsilon+\hbar \omega$, and $\varepsilon-\hbar \omega$ taken into account, we obtain 12 boundary conditions, allowing the calculation of all of the 12 unknown coefficients: $B_{0}, F_{0}, X_{1,2}, B_{ \pm}, F_{ \pm}$, and $X_{1,2}^{ \pm}$.

The coefficients $B_{0}, F_{0}$, and $X_{1,2}$ are given by the previous formulas (18a). For the remaining coefficients we obtain

$$
\begin{aligned}
X_{1}^{ \pm}+X_{2}^{ \pm}= & \pm \alpha\left[2 k\left(k-k_{ \pm}\right) g g_{ \pm}-i \delta_{ \pm}^{\prime}\left(g^{2}+k^{2}\right)\left(k-k_{ \pm}\right)\right. \\
& \left.-i \delta_{ \pm}^{\prime} \delta_{0}\left(k+k_{ \pm}\right)\right] / \delta_{0} \delta_{ \pm},
\end{aligned}
$$

$$
\begin{aligned}
& X_{1}^{ \pm} \exp \left(-g_{ \pm} w\right)+X_{2}^{ \pm} \exp \left(g_{ \pm} w\right) \\
& \quad= \pm \alpha\left[2 k\left(k-k_{ \pm}\right) g \delta_{ \pm}^{\prime}-i g_{ \pm}\left(g^{2}+k^{2}\right)\left(k-k_{ \pm}\right)\right. \\
& \left.\quad-i g_{ \pm} \delta_{0}\left(k+k_{ \pm}\right)\right] / \delta_{0} \delta_{ \pm}
\end{aligned}
$$

$$
F_{ \pm}= \pm \alpha\left\{i\left[2 k g \delta_{ \pm}-\delta_{0}\left(k+k_{ \pm}\right) g_{ \pm}\right]+2 k g \delta_{ \pm}^{\prime}\left(k-k_{ \pm}\right)\right.
$$$$
\left.-i\left(g^{2}+k^{2}\right) g_{ \pm}\left(k-k_{ \pm}\right)\right\} / \delta_{0} \delta_{ \pm} \text {, }
$$

$B_{ \pm}= \pm \alpha\left\{i\left[2 k \delta_{0}^{\prime} \delta_{ \pm}-\delta_{0}\left(k+k_{ \pm}\right) \delta_{ \pm}^{\prime}\right]+2 k g g_{ \pm}\left(k-k_{ \pm}\right)\right.$

$$
\left.-i\left(g^{2}+k^{2}\right) \delta_{ \pm}^{\prime}\left(k-k_{ \pm}\right)\right\} / \delta_{0} \delta_{ \pm},
$$

where

$$
\begin{aligned}
& \delta_{0}=g^{2} \Delta_{0}=\left(g^{2}-k^{2}\right) \sinh g w-2 i k g \cosh g w, \\
& \delta_{0}^{\prime}=g \cosh g w-i k \sinh g w, \\
& \delta_{ \pm}=\left(g_{ \pm}^{2}-k_{ \pm}^{2}\right) \sinh g_{ \pm} w-2 i k_{ \pm} g_{ \pm} \cosh g_{ \pm} w,
\end{aligned}
$$

and

$$
\delta_{ \pm}^{\prime}=g_{ \pm} \cosh g_{ \pm} w-i k_{ \pm} \sinh g_{ \pm} w .
$$

Using Eq. (11), we can find the current $j_{2}(x, t)$ :

$$
\begin{aligned}
j_{2}(x, t)= & (2 \hbar k / m)\left\{R_{+} \cos \left[\omega t-\left(k-k_{+}\right) x\right]\right. \\
& -I_{+} \sin \left[\omega t-\left(k-k_{+}\right) x\right]+R_{-} \cos \left[\omega t-\left(k_{-}-k\right) x\right] \\
& \left.-I_{-} \sin \left[\omega t-\left(k_{-}-k\right) x\right]\right\}
\end{aligned}
$$

where

$$
\begin{aligned}
R_{ \pm}= & \operatorname{Re}\left(F_{0}^{*} F_{ \pm}\right)= \pm\left(2 \alpha k g /\left|\delta_{0}\right|^{2}\right)\left(-2 k g+\left(1 /\left|\delta_{ \pm}\right|^{2}\right)\right. \\
& \times\left\{g_{ \pm}\left(k+k_{ \pm}\right)\left(g^{2}-k^{2}\right)\left(g_{ \pm}^{2}-k_{ \pm}^{2}\right) \sinh g w \sinh g_{ \pm} w\right. \\
& +4 k g k_{ \pm} g_{ \pm}^{2}\left(k+k_{ \pm}\right) \cosh g w \cosh g_{ \pm} w \\
& +\left(g^{2}+k^{2}\right)\left(k-k_{ \pm}\right) g_{ \pm}\left(g_{ \pm}^{2}+k_{ \pm}^{2}\right) \sinh g_{ \pm} w+2 k g \\
& \left.\left.\left(k-k_{ \pm}\right) k_{ \pm}\left[2 g_{ \pm}^{2} \cosh ^{2} g_{ \pm} w-\left(g_{ \pm}^{2}-k_{ \pm}^{2}\right) \sinh ^{2} g_{ \pm} w\right]\right\}\right),
\end{aligned}
$$

$$
\begin{aligned}
I_{ \pm}= & \operatorname{Im}\left(F_{0}^{*} F_{ \pm}\right)= \pm\left(\alpha k g / 2\left|\delta_{0}\right|^{2}\left|\delta_{ \pm}\right|^{2}\right)\left\{-2 g_{ \pm}\left(k+k_{ \pm}\right)\right. \\
& \times\left[k g\left(g_{ \pm}^{2}-k_{ \pm}^{2}\right) \sinh g_{ \pm} w \cosh g w-k_{ \pm} g_{ \pm}\right. \\
& \left.\times\left(g^{2}-k^{2}\right) \sinh g w \cosh g_{ \pm} w\right]+2 k g\left(k-k_{ \pm}\right) g_{ \pm} \\
& \times\left(g_{ \pm}^{2}-k_{ \pm}^{2}\right) \sinh g_{ \pm} w \cosh g_{ \pm} w+4 k g g_{ \pm} \\
& \times\left(k-k_{ \pm}\right) k_{ \pm}^{2} \sinh g_{ \pm} w \cosh g_{ \pm} w+2\left(g^{2}+k^{2}\right) \\
& \left.\times\left(k-k_{ \pm}\right) k_{ \pm} g_{ \pm}^{2} \cosh _{ \pm} g w\right\} .
\end{aligned}
$$

In the case of the thick barrier when the strong inequalities

$$
\exp (2 g w) \gg 1 \text { and } \exp \left(2 g_{ \pm} w\right) \gg 1
$$

take place, formulas (32)-(34) can be substantially simplified. Assume that as before we have

$$
\omega \ll \Omega, \Omega_{0}-\Omega .
$$

This allows us not to distinguish between $k$ and $k_{ \pm}$everywhere, excluding exponents, as well as between $g$ and $g_{ \pm}$. In this case $I_{+}$and $I_{-}$are negligibly small in comparison to $R_{+}$ and $R_{-}$, which are

$$
R_{ \pm} \cong \pm \frac{16 \alpha k^{2} g^{2}}{\left(g^{2}+k^{2}\right)^{2}} \exp (-g w)\left[\exp \left(-g_{ \pm} w\right)-\exp (-g w)\right]
$$

The inequalities (36) allow us to write

$$
\begin{aligned}
& k-k_{+} \cong k_{-}-k \cong(1 / 2) k(\omega / \Omega) \equiv q, \\
& g-g_{-} \cong g_{+}-g \cong(1 / 2) g\left[\omega /\left(\Omega_{0}-\Omega\right)\right] \equiv Q,
\end{aligned}
$$

and finally

$$
j_{2}(x, t)=-4 \alpha j_{0} \sinh Q w \cos (\omega t-q x) .
$$

By using formulas (31), it can be shown that in the case when the strong inequalities (35) are satisfied the current $j_{1}^{\prime}(x, t)$ is much smaller than $j_{2}(x, t)$. Therefore, we do not analyze it here. To obtain Eq. (40), we have used the conditions $\omega_{1} \ll \omega$ [Eq. (23)], $\exp (2 g w) \gg 1$ or $g w>2$ or 3 [Eq. (35)], $\omega \ll \Omega, \Omega_{0}-\Omega$ [Eq. (36)]. All of these conditions do not prohibit $Q w$ to be both $\ll 1$ and $\gg 1$. In the case when $Q w \ll 1$,

$$
j_{2}(x, t)=-j_{0}\left[g w \omega_{1} /\left(\Omega_{0}-\Omega\right)\right] \cos (\omega t-q x) .
$$

This result (for $x=0$ ) coincides with the quasistatic result (19) for $g w \gg 1$.

In the opposite case when

$$
\omega \gg 2\left(\Omega_{0}-\Omega\right) / g w>\omega_{1},
$$

we can observe a strong incompatibility of Eqs. (41) and (19) with each other, though the inequality (26) is securely satisfied. This means that the quasistatic approach fails. Let us note that the strong inequality in Eq. (42) is nothing but $\omega \tau_{T} \gg 1$ [see Eq. (1)]. In the case described by Eq. (42), the incorrect quasistatic approach predicts the much smaller current $j_{2}(x, t)$ than it is given by the correct formula (40).

\section{TUNNELING THROUGH A RECTANGULAR BARRIER WITH A TIME-DEPENDENT $\delta$-FUNCTION PERTURBATION}

In this section, we consider an inhomogeneously perturbed rectangular tunnel barrier (Fig. 3). We choose a $\delta$-function potential localized in the arbitrary position, $x=x_{1}$, within the barrier $\left(0 \leqslant x_{1} \leqslant w\right)$ as a time-dependent perturbation. Then the Schrödinger equation in the barrier acquires the following form: 


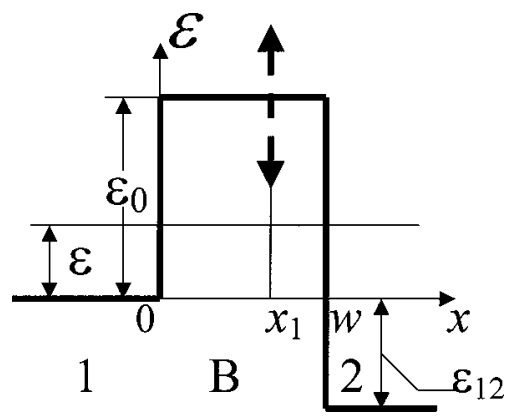

FIG. 3. Rectangular potential barrier with the time-dependent perturbation in the $\delta$-function form.

$$
i \hbar \frac{\partial \Psi_{B}}{\partial t}=-\frac{\hbar^{2}}{2 m} \frac{\partial^{2} \Psi_{B}}{\partial x^{2}}+\left[\varepsilon_{0}+a \delta\left(x-x_{1}\right) \cos \omega t\right] \Psi_{B}
$$

In comparison with Sec. III, we somewhat generalize our barrier model. We take into account that the electron effective masses $m_{1}=m_{2}$ in regions 1 and 2 (see Fig. 3) can differ from the barrier effective mass $m$ (such a situation corresponds to the structure considered in Ref. 16). We also foresee a possible lowering of the conduction band bottom in region 2 (as in Fig. 1), that is, $\varepsilon_{12} \neq 0$. Therefore new formulas for $F_{0}, B_{0}, X_{1,2}$ and stationary current $j_{0}$ are somewhat different from the analogous formulas in Sec. III [see Eqs. (18), (18a), and (18b)]. Specifically, we have

$$
\begin{aligned}
& F_{0}=-2 i \mu_{1} /\left[\left(1-\mu_{1} \mu_{2}\right) \sinh g w-i\left(\mu_{1}+\mu_{2}\right) \cosh g w\right], \\
& B_{0}=-\left[\left(1+i \mu_{1}\right) /\left(1-i \mu_{1}\right)\right]+\left[\left(1+i \mu_{2}\right) /\left(1-i \mu_{1}\right)\right] F e^{-g w},
\end{aligned}
$$

where $\mu_{1}=\left(k_{1} m / g m_{1}\right), \quad \mu_{2}=\left(k_{2} m / g m_{1}\right), k_{1}^{2}=2 m_{1} \varepsilon / \hbar^{2}$, and $k_{2}^{2}=2 m_{2}\left(\varepsilon+\varepsilon_{12}\right) / \hbar^{2}$. If $m_{1}=m$ and $\varepsilon_{12}=0$, we have $\mu_{1}=\mu_{2}$ $=\mu$, and Eqs. (44) and (45) coincide with the corresponding expressions for $F_{0}$ and $B_{0}$ in Eqs. (18a).

As in Sec. III, the case of the thick barrier $(g w \gg 1)$ is the most interesting. In this case

$$
\begin{aligned}
& F_{0} \cong-4 i \mu_{1} \exp (-g w) /\left[\left(1-i \mu_{1}\right)\left(1-i \mu_{2}\right)\right], \\
& B_{0}=-\left(1+i \mu_{1}\right) /\left(1-i \mu_{1}\right) .
\end{aligned}
$$

The stationary current component $j_{0}$ is

$$
j_{0}=4\left(\hbar k_{2} / m_{1}\right) \mu_{1}^{2} /|\Delta|^{2}
$$

with

$$
\begin{aligned}
|\Delta|^{2} & =\left(1-\mu_{1} \mu_{2}\right)^{2} \sinh ^{2} g w+\left(\mu_{1}+\mu_{2}\right)^{2} \cosh ^{2} g w \\
& \cong(1 / 4)\left(1+\mu_{1}^{2}\right)\left(1+\mu_{2}^{2}\right) e^{2 g w} .
\end{aligned}
$$

To obtain the transmitted nonstationary current component, we can use Eq. (11), in which we substitute

$$
F_{ \pm} \cong F_{0} g_{0} A_{ \pm} / g_{ \pm}
$$

where $g_{0}=-2 m a / \hbar^{2}$ and

$$
\begin{aligned}
A_{ \pm}= & {\left[\cosh g_{ \pm} x_{1}-i \mu_{(1 \pm)} \sinh g_{ \pm} x_{1}\right] /\left\{\left[1-\mu_{(1 \pm)} \mu_{(2 \pm)}\right]\right.} \\
& \left.\times \sinh g_{ \pm} w-i\left[\mu_{(1 \pm)}+\mu_{(2 \pm)}\right] \cosh g_{ \pm} w\right\}
\end{aligned}
$$

with $\mu_{(1,2 \pm)}=\left(k_{(1,2 \pm)} m / g_{ \pm} m_{1}\right)$. Formula (47) is approximate, because we obtain this expression by neglecting components proportional to $a^{2}, a^{3}$, and so on. Above, in Sec. III, we have produced the analogous neglects deriving both Eqs. (19) and (40). We have protected only the components proportional to $\varepsilon^{(1)}$ using the strong inequalities (21) and (23) as the graphic criteria.

As a result of substitution of the expression (47) in Eq. (11), we obtain

$$
\begin{aligned}
j_{1}(x, t)= & \left(\hbar / 2 m_{1}\right) g_{0}\left|F_{0}\right|^{2}\left\{[ ( k _ { 2 } + k _ { ( 2 + ) } ) / g _ { + } ] \left[A_{+}^{*} e^{i\left(q_{2} x-\omega t\right)}\right.\right. \\
& \left.+A_{+} e^{-i\left(q_{2} x-\omega t\right)}\right]+\left[\left(k_{2}+k_{(2-)}\right) / g_{-}\right]\left[A_{-}^{*} e^{-i\left(q_{2} x-\omega t\right)}\right. \\
& \left.\left.+A_{-} e^{i\left(q_{2} x-\omega t\right)}\right]\right\},
\end{aligned}
$$

where $q_{2}=(1 / 2) k_{2}(\omega / \Omega)$. In the case of the thick barrier formula (48) can be substantially simplified. Alongside the standard conditions $g w, g_{ \pm} w \gg 1$, we also assume $g_{ \pm} x_{1} \gg 1$. The $\delta$-function time-dependent perturbation should be placed sufficiently far from the cathode edge of the rectangular barrier. Then we have

$$
A_{ \pm} \cong\left(1-i \mu_{(2 \pm)}\right)^{-1} e^{-g_{ \pm}\left(w-x_{1}\right)} .
$$

Taking into account Eq. (39), we can obtain

$$
\begin{aligned}
j_{1}(x, t)= & 64\left(\hbar k_{2} / m_{1}\right)\left(g_{0} / g\right)\left[\mu_{1}^{2} /\left(1+\mu_{1}^{2}\right)\left(1+\mu_{2}^{2}\right)^{2}\right] \\
& \times e^{-3 g w+g x_{1}}\left\{\cosh \left[Q\left(w-x_{1}\right)\right] \cos \left(\omega t-q_{2} x\right)\right. \\
& \left.+\mu_{2} \sinh \left[Q\left(w-x_{1}\right)\right] \sin \left(\omega t-q_{2} x\right)\right\} \\
= & 4 j_{0}\left(g_{0} / g\right)\left(1+\mu_{2}^{2}\right)^{-1} e^{-g\left(w-x_{1}\right)}\left\{\cosh \left[Q\left(w-x_{1}\right)\right]\right. \\
& \times \cos \left(\omega t-q_{2} x\right)+\mu_{2} \sinh \left[Q\left(w-x_{1}\right)\right] \\
& \left.\times \sin \left(\omega t-q_{2} x\right)\right\} .
\end{aligned}
$$

Equation (50) demonstrates that the maximal "efficiency" of the high-frequency $\delta$-function modulation takes place if $x_{1}$ $=w$ and

$$
j_{1}(x, t)=4 j_{0}\left(g_{0} / g\right)\left(1+\mu_{2}^{2}\right)^{-1} \cosh Q w \cos \left(\omega t-q_{2} x\right) .
$$

Let us compare the formula (51) with the formula (40) from Sec. III. If $m=m_{1}$ and $\varepsilon_{12}=0$, they coincide in two limiting cases. At $Q w \ll 1$, they coincide if we assume that

$$
\alpha Q w=-g_{0} / g\left(1+\mu^{2}\right) \text {. }
$$

At $Q w \gg 1$, the coincidence takes place if

$$
\alpha=-g_{0} / g\left(1+\mu^{2}\right) \text {. }
$$

We see that the correlation between the value of $\varepsilon^{(1)}$ in Eq. (16) and the value $a$ in Eq. (43) depends on $Q w$. However, we observe the same tendency for $Q w>1$ in both considered cases: a drastic increase in the relation $j_{1} / j_{0}$ with an increase in the frequency $\omega$ and in the tunnel barrier thickness $w$.

\section{DISCUSSION}

A tunnel barrier as the modulated electron emitter, which emits electrons into a transit space, is considered above in a 
very simplified version, on the basis of a nonstationary wave function of a single electron that impinges on the barrier with a certain initial wave vector. If the tunnel transparence of the barrier is periodically modulated, such a barrier not only partially transfers and partially reflects the initial stationary electron wave but also becomes a source of the two oscillating waves with the frequency $\omega$, reflected and transferred, spreading on the different sides of the barrier. In the case of a thin barrier, these waves are comparable to each other in intensity, and even the reflected wave can exceed the transferred one. But in the case of a thick barrier [see Eq. (35)], the exponentially small transferred wave exceeds the reflected rival. The above-mentioned oscillating electron waves in the external space outside the barrier (in particular, the transferred wave in the transit space) form the basis for the design of the ballistic transit-time oscillator for the $\mathrm{THz}$ range. It is necessary to create an electric field in the transit space, which is coherent with the modulating potential in the barrier, and to select such a length of the transit space that the transferred wave in most of this space would be impeded by this electric field and, as a result, gain this field. Since the standing wavelength $\Lambda=\Lambda(\varepsilon)$ is energy dependent [is proportional to $\Omega / k \sim \sqrt{\Omega}$; see Eq. (13)], the barrier should be sufficiently thick: an energy width of an emitting electron beam localized around an electron Fermi energy in the cathode should be much narrower than this Fermi energy.

Considering an electron tunneling through a barrier with a time-dependent height in Sec. III, we can make sure that there exist two substantially different forms of a behavior that prevail over each other depending on the correlation between two frequencies: $\omega$ and $\omega_{1}=\varepsilon_{1} / \hbar=e V_{1} / \hbar$. Both these frequencies describe a high-frequency (HF) signal modulating a barrier height.

If $\omega \ll \omega_{1}$, we deal with the so-called quasistatic behavior of a HF current: this current does not depend on $\omega$. If the strong inequality $\omega_{1} \ll\left(\Omega_{0}-\Omega\right) / g w$ is realized, a linear and frequency-independent dependence of HF current amplitude on $\omega_{1}=e V_{1} / \hbar$ takes place. If the modulating signal is large, $\omega_{1} \geqslant\left(\Omega_{0}-\Omega\right) / g w$, the tunnel barrier demonstrates its nonlinear abilities and the HF current contains higher harmonics $(2 \omega, 3 \omega$, and so on) along with the initial harmonic $\omega$. Nevertheless, a harmonic composition of the HF current is independent of the value of $\omega$. Of course, we can expect that such a quasistatic behavior can be disrupted when the highest harmonics $n \omega$ with $n \gg 1$ reach and exceed $\omega_{1}=e V_{1} / \hbar$ and transfer from the quasistatic regime into a substantially nonstationary regime, which takes place at $\omega>\omega_{1}$.

If $\omega \gg \omega_{1}$, a linear and frequency-independent dependence of HF current amplitude on $\omega_{1}=e V_{1} / \hbar$ is restricted by the strong inequality $\omega \ll\left(\Omega_{0}-\Omega\right) / g w$. When a frequency $\omega$ is nearing $\left(\Omega_{0}-\Omega\right) / 2 g w$, an exponential growth of HF current amplitude with $\omega$ occurs. Along with amplitude of $j_{1}$, an exponential growth of a stationary component $\left(j_{0}\right)$ and higher harmonics takes place, but this growth is inhomogeneous for different components and harmonics. Initially, an increase in the first harmonic current $j_{1}$ leaves behind all the rest, and a small relation $j_{1} / j_{0}$ increases substantially and reaches a maximum value at $\omega \cong\left(\Omega_{0}-\Omega\right) / 2 g w$. But then the leadership transfers to the stationary component $j_{0}$, and the relation

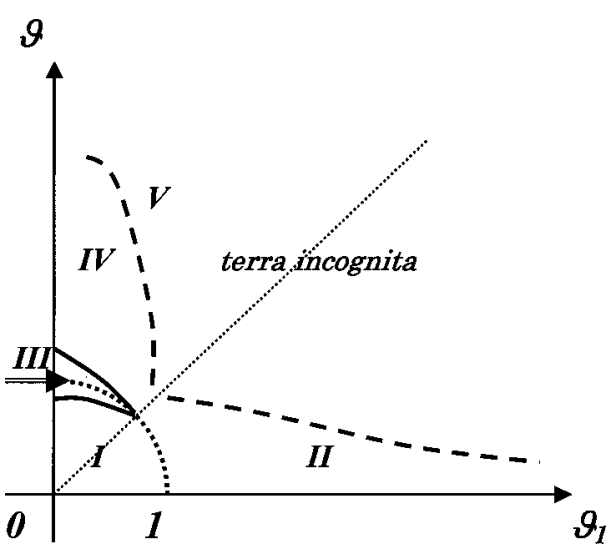

FIG. 4. Qualitative map of the different approximative results in the $\vartheta_{1} \vartheta$ plane for the rectangular tunnel barrier with the time-dependent height oscillating with a frequency $\omega$. Region I: quasistatic linear "conductance." Region II: quasistatic nonlinear "conductance." Region III: quasiresonant growth of a nonstatic linear "conductance." Region IV: nonstatic regime with a single-photon absorption. Region V: nonstatic regimes with a twoand a more than two-photon absorption.

$j_{1} / j_{0}$ decreases after passing the maximum. An increase in $j_{0}$ for thick tunnel barriers $(g w \gg 1)$ with increasing $\omega$ is well known $^{3-6}$ and explained by an absorption of the HF photon, $\hbar \omega$, during the electron tunneling. The larger the value of $\hbar \omega$ and the thicker the barrier, the more effective the result of such intrabarrier absorption can be observed on the background of an exponentially small initial tunnel current.

Operating by the same terminology, the above-described increase in the first harmonic current $j_{1}$ could be attributed to a virtual absorption of the HF photon, $\hbar \omega$, by the tunneling electron. Such an electron absorbs the photon during the tunneling, passes a part of the tunnel path having the exceeded energy $\varepsilon+\hbar \omega$, and emits this $\hbar \omega$-excess before leaving the barrier.

It is known that a further increase in the effective barrier thickness $g w$ leads to domination of multiphoton processes in the tunneling through the barrier (with absorption of two, three, and more photons: see Ref. 6 where an approximate evaluation of such processes is presented in detail). In Fig. 4 we have tried to show a certain map of tunneling regimes for the rectangular barrier with a time-dependant height experiencing small oscillations of the frequency $\omega$. This map is constructed in the dimensionless coordinates $\vartheta_{1}$ $=\omega_{1} g w /\left(\Omega_{0}-\Omega\right)$ and $\vartheta=\omega g w /\left(\Omega_{0}-\Omega\right)$, and it is assumed that $\omega, \omega_{1} \ll \Omega_{0}-\Omega$. A linear quasistatic regime when a tunnel current $j_{1}$ is connected with the "voltage" $V_{1}=\hbar \omega_{1} / e$ by a linear "conductivity," which depends on neither $\omega$ nor $\omega_{1}$, takes place in region I where $\omega, \omega_{1} \ll\left(\Omega_{0}-\Omega\right) / g w$. Region II, which is positioned on the right side where $\omega \ll \omega_{1}$, corresponds to quasistatic nonlinear regimes, for which the tunnel barrier behaves in relation to the voltage $V_{1}$ as a static nonlinear conductor. A tunnel current is a composition of different harmonics of the initial frequency: $\omega, 2 \omega, 3 \omega$, and so on. This harmonic composition depends on $\omega_{1}$ but is independent of $\omega$. We can assume that this composition gets richer with an increase in $\omega_{1}$.

The opposite strong inequality, $\omega \gg \omega_{1}$, leads to substantially nonstatic tunnel regimes when a tunneling electron can 
absorb one or more photons of the HF electric field connected with the HF voltage $V_{1}$. This absorption results in a very substantial growth of the stationary tunnel current $j_{0}$. Boundaries between region IV where the single-photon absorption dominates and region $\mathrm{V}$ with the dominating twophoton (or more-than-two-photon) absorption are indefinite because they probably do not depend only on the variables $\vartheta$ and $\vartheta_{1}$. There is a narrow region III between regions I and IV where the above-described quasiresonant increase in $j_{1} / j_{0}$, which is the basic result of our work, takes place. All the remaining area in Fig. 4 is now a terraincognita where a multiphoton absorption competes with a quasistatic emission of higher current harmonics.

\section{CONCLUSION}

It is shown that a high-frequency modulation of a tunnelbarrier height leads not only to the well-known growth of a tunnel transparence for a stationary tunnel current ${ }^{3-6}$ but also to an increased amplitude of a high-frequency tunnel current $j_{1}$ entering region 2 (a transit space) on the right side of the tunnel barrier (Fig. 2). Such an effect occurs for sufficiently thick tunnel barriers $(g w \gg 1)$, small amplitudes of the modulating voltage $V_{1}=\hbar \omega_{1} / e(\ll \hbar \omega / e)$, and intermediate frequencies of this modulation $\left[\omega \sim\left(\Omega_{0}-\Omega\right) / 2 g w\right]$. This relative growth of the modulation-induced HF current is not described by the quasistatic theory. In the indicated intermediate frequency region, the stationary tunnel current component $j_{0}$ increases much more slowly than the $j_{1}$ component. Evidently, this effect can be used to increase an efficiency of ballistic tunnett diodes.

The results obtained in the case of homogeneous oscillations of the height of the rectangular tunnel barrier have been compared with the results for another form of the timedependent perturbation: the oscillating $\delta$ function positioned inside the barrier. We have revealed that only the disposition of the $\delta$-function perturbation near the anode side of the barrier $\left(x_{1}=w\right)$ allows one to reach a substantial modulation of the tunnel current.

\section{ACKNOWLEDGMENTS}

The authors thank Dr. N. Vagidov for the friendly assistance and Dr. S. Tipton for the fruitful comments. Z.S.G. thanks professor M. I. Dykman for numerous discussions. This work was supported by the AFOSR through the MURI program, Grant No. F 4960-00-0328.

${ }^{1}$ L. V. Keldysh, Zh. Eksp. Teor. Fiz. 47, 1945 (1964) [Sov. Phys. JETP 20, 1307 (1965)].

${ }^{2}$ L. D. Landau and E. M. Lifshitz, Quantum Mechanics (Pergamon, New York, 1977).

${ }^{3}$ M. Büttiker and R. Landauer, Phys. Rev. Lett. 49, 1739 (1982).

${ }^{4}$ R. Landauer and Th. Martin, Rev. Mod. Phys. 66, 217 (1994).

${ }^{5}$ B. I. Ivlev and V. I. Mel'nikov, Phys. Rev. Lett. 55, 1614 (1985).

${ }^{6}$ B. I. Ivlev and V. I. Mel'nikov, Sov. Phys. JETP 90, 2208 (1986).

${ }^{7}$ S. D. Ganichev, I. N. Yassievich, W. Prettl, J. Diener, B. K. Meyer, and K. W. Benz, Phys. Rev. Lett. 75, 1590 (1995).

${ }^{8}$ S. D. Ganichev, E. Ziemann, Th. Gleim, W. Prettl, I. N. Yassievich, V. I. Perel, I. Wilke, and E. E. Haller, Phys. Rev. Lett. 80, 2409 (1998).

${ }^{9}$ E. Ziemann, S. D. Ganichev, W. Prettl, I. N. Yassievich, and V. I. Perel, J. Appl. Phys. 87, 3843 (2000).

${ }^{10}$ S. D. Ganichev, I. N. Yassievich, V. I. Perel, H. Ketterl, and W. Prettl, Phys. Rev. B 65, 085203 (2002).

${ }^{11}$ M. Shur, Physics of Semiconductor Devices (Prentice, Englewood Cliffs, NJ, 1990).

${ }^{12}$ S. M. Sze, Physics of Semiconductor Devices (Wiley, New York, 1981).

${ }^{13}$ P. Plotka, J. Nishizawa, T. Kurabayashi, and H. Makabe, IEEE Trans. Electron Devices 50, 867 (2003).

${ }^{14}$ Z. S. Gribnikov, N. Z. Vagidov, V. V. Mitin, and G. I. Haddad, J. Appl. Phys. 93, 5435 (2003).

${ }^{15}$ Z. S. Gribnikov, N. Z. Vagidov, V. V. Mitin, and G. I. Haddad, Physica E (Amsterdam) 19, 89 (2003).

${ }^{16}$ Z. S. Gribnikov, N. Z. Vagidov, and G. I. Haddad, J. Appl. Phys. 95, 1489 (2004). 